\title{
SEQUENTIAL CLASSIFICATION OF ACTIVE SONAR RETURNS
}

\author{
Roger F. Dwyer and Ronald P. Radlinski \\ Naval Undersea Warfare Center \\ New London, CT 06320
}

\begin{abstract}
tial classifier is demonstrated by simulation. Four typical sonar classes representing rigid and elastic spheres of different materials are simulated. The results which demonstrate the advantage of a deferred decision strategy are displayed for two performance criteria in a confusion matrix extended to include a decision pending category.
\end{abstract}

An automated active sonar classifier is representation[1,2,3]. Both the ambiguity designed based on the principle of deferred function and Wigner distribution function decision. Given the loglikelihoods of all are examples time-frequency representaclasses up to the current return a clssifica- tions. Here the fourth-order cumulant spection decision is deferred until the next re- trum will be used to extract information turn if certain performance criteria are not about the targets to be classified. Four satisfied. This active sonar sequential clas- classes of targets are considered. Three are sification procedure has the advanage of re- spherical with the same radius. The mateducing errors and minimizing the number of rial of the spheres are either steel or lucite. returns required to reach a correct decision. Both rigid and elastic modes of the spheres The performance of the active sonar sequen- are considered. The fourth class is noise.

\section{INTRODUCTION}

In active sonar, a return from a target is often analyzed in a time-frequency

The classification model employed assumes a two-dimensional grid of frequency components from a slice of the fourth-order cumulant spectrum. For subsequent returns the model allows the components of the fourth-order cumulant spectrum to be correlated with previous corresponding components.

\section{SONAR MODEL}

The active sonar data model assumed here uses the output of the fourth-order cumulant spectrum[4],

This work was supported by the IR Program of NUWC and by ONT under the NU3B Program at $N U W C$.

$$
C_{4}\left(\omega_{1}, \omega_{2}, \omega_{3}\right)=
$$




$$
\int_{-\infty}^{\infty} \int_{-\infty}^{\infty} \int_{-\infty}^{\infty} c_{4}\left(\tau_{1}, \tau_{2}, \tau_{3}\right) d \tau_{1} d \tau_{2} d \tau_{3}
$$

where, $c_{4}\left(\tau_{1}, \tau_{2}, \tau_{3}\right)$, is the fourth-order cumulant. Details of the fourth-order cumulant and spectrum can be found in[5]. Here, a special case will be used to extract information from the impulse response of a rigid sphere, a thin elastic steel sphere, and a thin elastic lucite sphere. The impulse reponses of the spheres were obtained from a program developed by J. Patel from NUWC, based on Junger and Feit[6].

Classification is based on the components of the fourth-order cumulant spectrum. For each return a set of components are extracted and sent to the sequential classifier. In general all components of the fourth-order cumulant spectrum could be extracted and used for classification. But this would require a significant processing capability. Here a judicious selection of components were extracted and used in the classifier. Future work will include all components in the classifier.

The fourth-order cumulant spectra for the spheres are shown below:

(1) rigid sphere, designated as class 1 ,

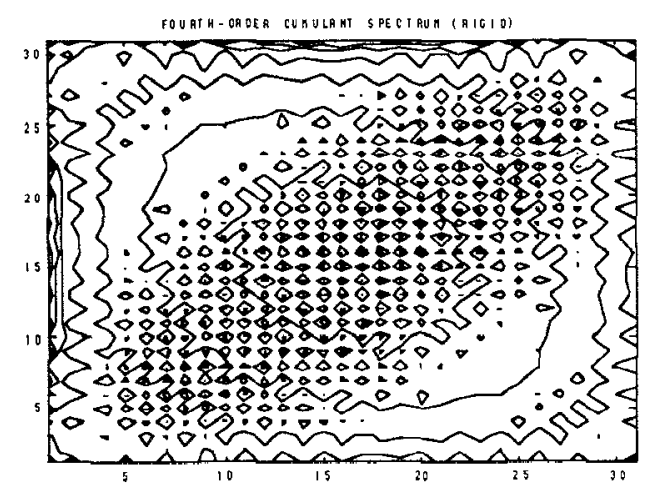

(2) thin elastic steel sphere, designated as class 2 ,

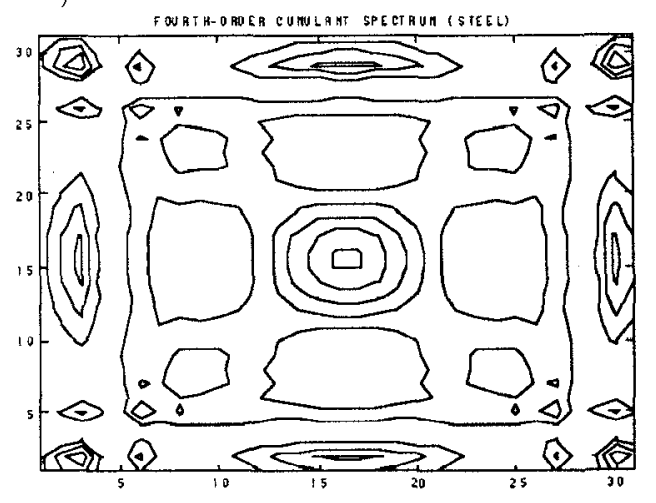

(3) thin elastic lucite sphere, designated as class 3 ,

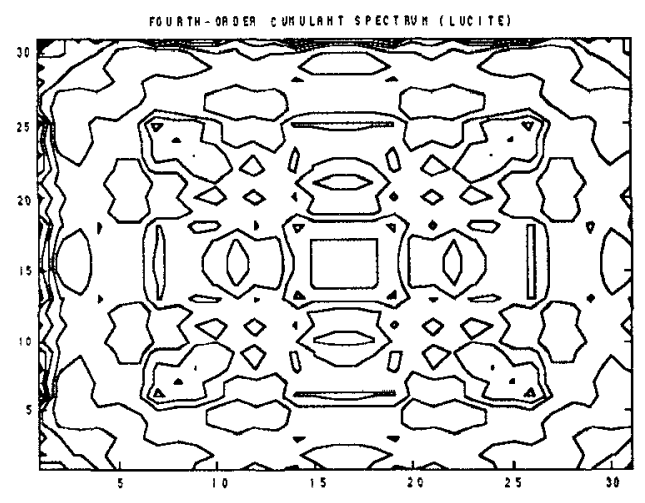

(4) noise, designated as class 0 ,

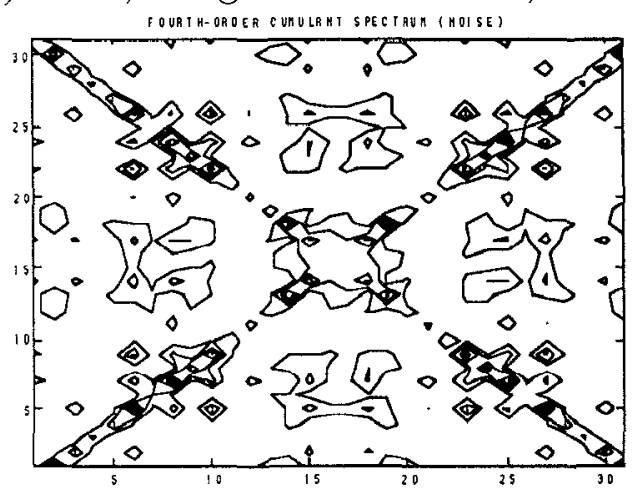

The axes for each figure are in normalized frequency. Since the patterns depicted above are all distinctive, a classifier properly encoded when presented with the data 
from one class could in principle decide correctly. In practice noise and resolution will limit performance. Here, only a small number of samples from each class are used in the classification simulation to demonstrate the method. Actually, two simulations were performed with the data. In the first case the data were used as given. In the second case the data were compressed or normalized. This case proved to be difficult for the classifier because of the reduced dynamic range of the data. The symmetry in the patterns above appear to aid identification. But samples from only one octant in each fourthorder cumulant spectrum were chosen to encode the sequential classifier.

\section{ACTIVE SONAR SEQUENTIAL CLASSIFICATION}

Since the fourth-order cumulant spec- where, trum is in general a complex function, its components can be represented by two multidimensional probability density functions. To simplify the analysis without losing generality only the real part of the output of the fourth-order cumulant spectrum will be considered. Therefore, the components of the fourth-order cumulant spectrum will be represented by a multidimensional Gaussian density function over all returns. This assumption could perhaps be extended to nonGaussian densities by utilizing mixtures of Gaussians[7]. But this will be left to future work.

For each class the information from a set of components of the fourth-order cumulant spectrum are encoded into a covariance matrix of a multidimensional Gaussian density function. This is done by letting the square of the sample values represent the variances in the covariance matrices. For the data given above the samples were assumed independent and to have zero mean. However, the classifier can encode correlated and nonzero mean samples in more general cases. Given a sequence of returns the sequential classifier decides which class is present based on the covariance matrices representing the classes.

Since each class is modeled as a multidimensional Gaussian process, the loglikelihood ratio of two classes is,

$$
\begin{gathered}
\Lambda_{n p q}(\underline{X})=\left\{\frac{1}{2} \ln \left[\frac{\left|M_{q}\right|}{\left|M_{p}\right|}\right]\right. \\
\left.-\frac{1}{2} \underline{X^{T}}\left(M_{p}^{-1}-M_{q}^{-1}\right) \underline{X}\right\},
\end{gathered}
$$

$$
\begin{gathered}
\underline{X}= \\
{\left[X_{11}(1), X_{12}(1), \ldots, X_{M D}(1), \ldots, X_{M D}(n)\right],}
\end{gathered}
$$

is a vector representing the components of the fourth-order cumulant spectrum for each return, $p, q=0,1,2,3, p \neq q$, represent the four classes, and, $n$ is a random variable representing the number of returns required to make a decision. The matrices of (3.1) are defined as,

$$
M_{m}=E\left[\underline{X_{k j}} \underline{X_{k j}^{T}}\right],
$$

for, $m=0,1,2,3$, over all returns. These results can easily be extended to higher dimensions.

The performance of the sequential classifier for each loglikelihood ratio is based on the operating characteristic function (OCF) and the average sample number (ASN) as 
defined by Wald $[8,9,10,11]$. The OCF is the in the performance matrix. For both cases probability, $L(h)$, of accepting $H_{q}$, defined five independent trials were run. The peras,

$$
L(h)=\left(A^{h}-1\right) /\left(A^{h}-B^{h}\right),
$$

formance matrix for each return gives the average of the five trials. To demonstrate the effect of deferred decision two thresholds settings, $(-.5, .5)$ and $(-2.1972,2.1972)$, reprewhere, $\left(H_{p}: h=-1, H_{q}: h=1\right), \mathrm{A}$ and $\mathrm{B}$ are upper and lower thresholds, respectively. For the loglikelihood ratios the upper and lower thresholds are, respectively,

$$
\begin{aligned}
& a=\ln (A)=\ln \left(\frac{1-\beta}{\alpha}\right), \\
& b=\ln (B)=\ln \left(\frac{\beta}{1-\alpha}\right) .
\end{aligned}
$$

The parameters, $\alpha$, and, $\beta$, represent the false alarm and false dissmissal probabilities, respectively. If $\Lambda_{n p q} \geq a, H_{p}$ is decided, if, $\Lambda_{n p q} \leq b, H_{q}$ is decided. Whereas, if, $b<\Lambda_{n p q}<a$, it is not used in the decision at this return. In the simulations, $\alpha=\beta$, therefore, $b=-a$. Two sets of thresholds will be considered in the simulations.

\section{RESULTS}

In the simulations four hypotheses or classes representing the data discussed in section 2 will be considered. In the figures, 0 will represent, $H_{0}, 1$ will represent, $H_{1}$, 2 will represent, $H_{2}$, and 3 will represent, $H_{3}$. The classifier considers simultaneously all independent pairs of classes for a total of 6 . The performance is summarized in a matrix called the performance matrix. The performance matrix compares the classification performance for each class as a function of the number of returns. Since the classifier is sequential a classification may not be made at each return. To account for this a decision pending (DP) category is included figure. 
THIRD RETURN (CASE 1)

\begin{tabular}{|c|c|c|c|c|c|c|c|c|c|c|c|}
\hline \multicolumn{3}{|c|}{$\begin{array}{l}n=3 \\
\text { sphere }\end{array}$} & \multicolumn{3}{|c|}{$\begin{array}{l}a=.5 \\
b=-.5\end{array}$} & \multicolumn{3}{|c|}{$\begin{array}{l}n=3 \\
\text { sphere }\end{array}$} & \multicolumn{3}{|c|}{$\begin{array}{l}a=2.1972 \\
b=-2.197\end{array}$} \\
\hline & 0 & 1 & 2 & 3 & $\mathrm{DP}$ & & 0 & 1 & 2 & 3 & DP \\
\hline 0 & 1 & 0 & 0 & $\mathbf{0}$ & 0 & 0 & 1 & 0 & 0 & 0 & $0^{\prime}$ \\
\hline 1 & 0 & $4 / 5$ & 0 & 0 & $1 / 5$ & 1 & 0 & $3 / 5$ & 0 & 0 & $2 / 5$ \\
\hline 2 & 0 & 0 & 1 & 0 & 0 & 2 & 0 & 0 & $2 / 5$ & 0 & $3 / 5$ \\
\hline 3 & 0 & 0 & 0 & 1 & 0 & 3 & 0 & 0 & 0 & 1 & 0 \\
\hline
\end{tabular}

Again, there are no missclassifications and deferred decisions occur more often in the right figure.

FOURTH RETURN (CASE 1)

\begin{tabular}{|c|c|c|c|c|c|c|c|c|c|c|c|}
\hline \multicolumn{3}{|c|}{$\begin{array}{l}n=4 \\
\text { sphere }\end{array}$} & \multicolumn{3}{|c|}{$\begin{array}{l}a=.5 \\
b=-5\end{array}$} & \multicolumn{3}{|c|}{$\begin{array}{l}n=4 \\
\text { sphere }\end{array}$} & \multicolumn{3}{|c|}{$\begin{array}{l}a=2.1972 \\
b=-2.197\end{array}$} \\
\hline & 0 & 1 & 2 & 3 & DP & & 0 & 1 & 2 & 3 & DP \\
\hline 0 & 1 & 0 & 0 & 0 & 0 & 0 & 1 & $\overline{0}$ & 0 & 0 & 0 \\
\hline 1 & 0 & $4 / 5$ & 0 & $1 / 5$ & 0 & 1 & 0 & $3 / 5$ & 0 & 0 & $2 / 5$ \\
\hline 2 & 0 & 0 & 1 & 0 & 0 & 2 & 0 & 0 & $3 / 5$ & 0 & $2 / 5$ \\
\hline 3 & 0 & 0 & 0 & 1 & 0 & 3 & 0 & 0 & 0 & 1 & 0 \\
\hline
\end{tabular}

There's one missclassification in the left figure but none in the right. At the fifth and following returns all classes are correctly classified.

\section{CASE 2 NORMALIZED DATA:}

Classification in the normalized data case required many more returns. Some classes were not decided even at the twentyfifth return. The right figures have fewer misclassifications and more deferred decisions. But errors in classification occur in both left and right figures.
FIRST RETURN (CASE 2)

\begin{tabular}{|c|c|c|c|c|c|c|c|c|c|c|c|}
\hline \multicolumn{6}{|c|}{$\begin{array}{ll}n=1 & a=.5 \\
\text { sphere }(N) \quad b=-5\end{array}$} & \multicolumn{4}{|c|}{$\begin{array}{l}n=1 \\
\text { sphere }(N)\end{array}$} & \multicolumn{2}{|c|}{$\begin{array}{l}a=2.1972 \\
b=-2.197\end{array}$} \\
\hline & 0 & 1 & 2 & 3 & DP & & 0 & 1 & 2 & 3 & $\mathrm{DP}$ \\
\hline 0 & $1 / 5$ & 0 & 0 & 0 & 4/3 & 0 & 0 & 0 & 0 & 0 & 1 \\
\hline 1 & $2 / 5$ & 0 & 0 & $1 / 5$ & & 1 & 0 & 0 & 0 & 0 & 1 \\
\hline 2 & $3 / 5$ & $\overline{0}$ & 0 & 0 & $2 / 5$ & 2 & 0 & 0 & 0 & 0 & 1 \\
\hline 3 & $2 / 5$ & 0 & 0 & $1 / 5$ & $2 / 5$ & 3 & 0 & 0 & 0 & 0 & 1 \\
\hline
\end{tabular}

There are several missclassifications in the left figure but all decisions are deferred until the next return in the right.

\section{FIFTEENTH RETURN (CASE 2)}

\begin{tabular}{|c|c|c|c|c|c|c|c|c|c|c|c|}
\hline \multicolumn{3}{|c|}{$\begin{array}{l}n=15 \\
\text { sphere }(N)\end{array}$} & \multicolumn{3}{|c|}{$\begin{array}{l}a=.5 \\
b=-.5\end{array}$} & \multicolumn{3}{|c|}{$\begin{array}{l}n=15 \\
\text { sphere }(\mathrm{N})\end{array}$} & \multicolumn{3}{|c|}{$\begin{array}{l}a=2.1972 \\
b=-2.197\end{array}$} \\
\hline & 10 & 1 & 2 & 3 & DP & & 0 & 1 & 2 & 3 & DP \\
\hline 0 & 1 & 0 & 0 & 0 & 0 & 0 & 1 & 0 & 0 & 0 & 0 \\
\hline 1 & 0 & $4 / 5$ & $1 / 5$ & 0 & 0 & 1 & $1 / 5$ & $1 / 5$ & 0 & $\overline{0}$ & $3 / 5$ \\
\hline 2 & $1 / 5$ & 0 & $4 / 5$ & 0 & 0 & 2 & $1 / 5$ & 0 & $3 / 5$ & 0 & $1 / 5$ \\
\hline 3 & 0 & 0 & 0 & 1 & 0 & 3 & 0 & 0 & 0 & 1 & 0 \\
\hline
\end{tabular}

Errors in classification are in both figures.

TWENTYFIFTH RETURN (CASE 2)

\begin{tabular}{|c|c|c|c|c|c|c|c|c|c|c|c|}
\hline \multicolumn{3}{|c|}{$\begin{array}{l}n=25 \\
\text { sphere (N) }\end{array}$} & \multicolumn{3}{|c|}{$\begin{array}{l}a=.5 \\
b=-.5\end{array}$} & \multicolumn{3}{|c|}{$\begin{array}{l}n=25 \\
\text { sphere }(\mathrm{N})\end{array}$} & \multicolumn{3}{|c|}{$\begin{array}{l}a=2.1972 \\
b=-2.197\end{array}$} \\
\hline & 0 & 1 & 2 & 3 & $\mathrm{DP}$ & & \begin{tabular}{|l|l|}
0 \\
\end{tabular} & 1 & \begin{tabular}{|l|l|}
2 \\
\end{tabular} & 3 & $\mathrm{DP}$ \\
\hline 0 & 1 & 0 & 0 & 0 & 0 & 0 & 1 & $\overline{0}$ & 0 & 0 & 0 \\
\hline 1 & 0 & $4 / 5$ & $1 / 5$ & 0 & 0 & 1 & 0 & $3 / 5$ & $1 / 5$ & 0 & $1 / 5$ \\
\hline 2 & $1 / 5$ & 0 & $4 / 5$ & 0 & 0 & 2 & $1 / 5$ & 0 & $3 / 5$ & 0 & $1 / 5$ \\
\hline 3 & 0 & $\overline{0}$ & 0 & 1 & 0 & 3 & 0 & 0 & 0 & 1 & 0 \\
\hline
\end{tabular}

Even at the twentyfifth return errors occur in both figures. However, less errors occur in the right figure because of the deferred decision strategy. 


\section{CONCLUSIONS}

Information extracted from rigid and elastic spheres by the fourth-order cumulant spectrum were used to encode an automated active sonar sequential classifier. The sequential classifier operated under the principle of deferred decision. If at a particular return classification criteria were not satisfied a decision would be deferred. By deferring decisions, classification errors were shown to be controllable. Two simulations from the data representing rigid and elastic spheres demonstrated the deferred decision strategy. Specifically, by postponing a classification decision until the next return missclassification errors were reduced.

\section{ACKNOWLEDGMENT}

The authors acknowledge the assistance of Dr. Theresa Baus in generating the spherical test data.

\section{REFERENCES}

1. Nai-chyuan Yen, Louis R. Dragonette, and Susan K. Numrich, "Time-Frequency Analysis of Acoustic Scattering from Elastic Objects," J. Acoust. Soc. Am. 87(6), pp 2359-2370, June 1990.

2. Nai-chyan Yen, "Time-Frequency Display of Sonar Signal," IEEE Proceedings of Oceans'91, pp 1446-1451, October 1-3, 1991, Honolulu, Hawaii.

3. A. Nuttall, "Alias-Free Smoothed Wigner Distribution Function for Discrete-Time Samples," IEEE Proceedings of Oceans'91, pp 1438-1445, October 1-3, 1991, Honolulu,
Hawaii.

4. R. Dwyer, "Sonar Processing With Higher-Order Methods," IEEE Proceedings of Oceans'91, pp 1452-1459, October 1-3, 1991, Honolulu, Hawaii.

5. D. Brillinger and M. Rosenblatt, "Asymptotic Theory of Kth-Order Spectra," In: Spectral Analysis of Time Series (B. Harris, ed.), 1967, pp 153-188, Wiley, New York.

6. M. Junger and D. Feit, Sound Structures and Their Interaction, The MIT Press 1972.

7. R. Dwyer and L. Kurz, "Characterizing Partition Detectors With Stationary and Quasi-Stationary Markov Dependent Data," IEEE Transactions on Information Theory, Vol. II-32, No. 4, July 1986.

8. A. Wald, Sequential Analysis, John Wiley and Sons, Inc., New York 1947.

9. R. Dwyer and F. McMullen, "Sonar Detection With Correlated Returns," IEEE Proceedings of Oceans'91, pp 1570-1574, October 1-3, 1991, Honolulu, Hawaii.

10. L. C. Palmer, "Sequential Tests to Select Among M Hypotheses," IEEE Trans. on IT, Vol. IT-18, no. 1, pp 211-214, January 1972.

11. I. Jouny and F. Garber, "Design and Comparison of M-ary Sequential Hypothesis Tests for Automatic Target Recognition," To Appear in IEEE Trans. on AES. 